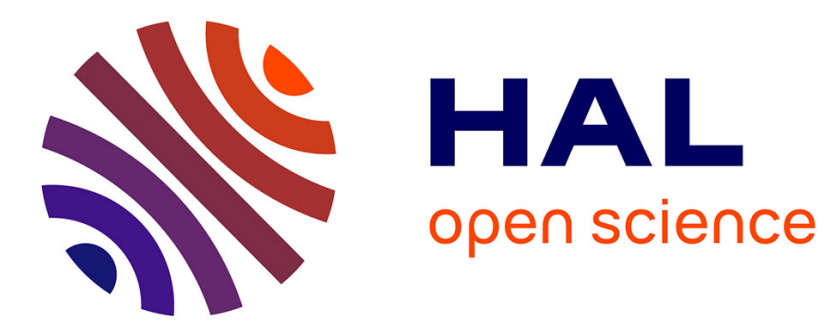

\title{
Ionisation de deutons polarisés dans un cyclotron par une source à arc de grande intensité
}

\author{
R. Maillard, A. Papineau
}

\section{To cite this version:}

R. Maillard, A. Papineau. Ionisation de deutons polarisés dans un cyclotron par une source à arc de grande intensité. Revue de Physique Appliquée, 1969, 4 (2), pp.175-175. 10.1051/rphysap:0196900402017500 . jpa-00243203

\section{HAL Id: jpa-00243203 https://hal.science/jpa-00243203}

Submitted on 1 Jan 1969

HAL is a multi-disciplinary open access archive for the deposit and dissemination of scientific research documents, whether they are published or not. The documents may come from teaching and research institutions in France or abroad, or from public or private research centers.
L'archive ouverte pluridisciplinaire HAL, est destinée au dépôt et à la diffusion de documents scientifiques de niveau recherche, publiés ou non, émanant des établissements d'enseignement et de recherche français ou étrangers, des laboratoires publics ou privés. 


\title{
IONISATION DE DEUTONS POLARISÉS DANS UN GYGLOTRON PAR UNE SOURGE A ARG DE GRANDE INTENSITÉ
}

\author{
R. MAILLARD et A. PAPINEAU, \\ Service de Physique Nucléaire à Moyenne Énergie, C.E.N., Saclay.
}

\begin{abstract}
Résumé. - Au cyclotron à fréquence fixe de Saclay, un faisceau de 5 nA de deutons polarisés a été obtenu en ionisant les deutons au moyen d'une source à arc à cathode de grande surface. Dans ces conditions, le taux de polarisation est de $50 \%$.
\end{abstract}

Abstract. - With an ionization source with a large cathode, the beam of polarized deuterons now has an intensity of $5 \mathrm{nA}$. In these conditions, the polarization yield is estimated at $50 \%$.

Le faisceau atomique de la source de deutons polarisés $[1,2,3,4,5]$ a été initialement ionisé au centre du cyclotron à fréquence fixe par une source de type magnéton inverse [6]. Le faisceau externe était de $2 \times 10^{8}$ particules environ.

I. Ionisation par source à arc. - Une source d'ions multichargée avec support de gaz [7] était utilisée depuis plusieurs années pour obtenir un faisceau externe de $50 \mu \mathrm{A}$ de $\mathrm{He}^{++}$. Il a semblé intéressant d'utiliser cette technique pour améliorer l'intensité du faisceau de deutons polarisés. L'anode de cette source à large cathode (1 $\mathrm{cm}^{2}$ environ) a été percée de part en part pour permettre le passage du faisceau atomique sans compression à l'intérieur de celle-ci. Le gaz utilisé a été $\mathrm{CO}_{2}$, qui a permis d'augmenter le moins possible la pression à l'intérieur de la chambre du cyclotron. En effet, la distance de 2 mètres entre la sortie de la source de deutons polarisés et le centre du cyclotron est très grande et il est indispensable de réduire au minimum la pression à l'intérieur de la chambre du cyclotron afin d'empêcher la diffusion multiple du faisceau atomique. La tension de l'arc était de 320 volts et son intensité de 10 ampères. Dans ces conditions, l'intensité du faisceau externe de deutons polarisés a été portée à 5 nanoampères.

II. Mesure de la polarisation du faisceau. - Jusqu'à présent, aucune mesure absolue de polarisation du faisceau de deutons n'a été faite à l'énergie utilisée $(21,8 \pm 0,2 \mathrm{MeV})$. Pour estimer le taux de polarisation du faisceau, des mesures sont faites en fonctionnement et immédiatement après coupure de l'aimant sextupolaire Stern-Gerlach de la source de deutons polarisés. Dans les conditions expérimentales précédentes, le taux de polarisation a été estimé à $0,8 \pm 0,07$. Avec la source à arc, la polarisation a été estimée de la même manière à $0,5 \pm 0,07$.

De plus, de nombreuses expériences ont été faites précédemment en utilisant un polarimètre à carbone à cible épaisse (60 mg environ). L'énergie du faisceau au centre de la cible après passage dans divers absorbeurs était de 20,6 MeV au lieu de 21,8 MeV d'énergie incidente. Dans ces conditions, pour un faisceau incident de polarisation 0,8 (source magnétron), l'asymétrie plane vectorielle à $45^{\circ} \pm 3^{\circ}$ était de $16,5 \%$ (angle de détection horizontal) et l'asymétrie tensorielle à $27^{\circ} \pm 2^{\circ}$ était de $14,5 \%$ (angle de détection vertical) pour la réaction ${ }^{12} \mathrm{C}(\mathrm{d}, \mathrm{p}){ }^{12} \mathrm{C}$ (fondamental). Les polarisations mesurées dans les mêmes conditions avec la source à arc étaient respectivement de 11,5\% et $9,2 \%$.

En attendant d'avoir un point de polarisation absolue pour le faisceau, l'emploi de la source à arc permet de multiplier par plus de 100 l'intensité du faisceau tout en conservant une polarisation moitié de celle donnée par la source de deutons polarisés.

\section{BIBLIOGRAPHIE}

[1] Thirion (J.), Beurtey (R.) et Papineau (A.), Helv. Phys. Acta, 1960, suppl. 6, 108.

[2] Beurtey (R.), Papineau (A.) et Thirion (J.), Nuovo Cimento, 1961, 19, 207.

[3] Thirion (J.), Beurtey (R.) et Papineau (A.), J. Physique Rad., 1961, 22, suppl. no 11, 141 A.

[4] Beurtey (R.), Chaminade (R.), Falcoz (A.), MAIl_LARD (R.), Mikumo (T.), Papineau (A.),
Schecter (L.) et Thirion (J.), J. Physique Rad. 1963, 24, 1038.

[5] BeURTey (R.), Experientia, 1965, suppl. 12, 33.

[6] Garreti (D.), BenEZECh (P.) et KNITTiNER (G.), NuclInstr. Methods, 1962, 17, 123.

[7] Papineau (A.), Benezech (P.) et Mailitard (R.), J. Physique Rad., 1960, 21, 410. 